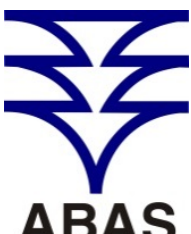

ABAS

ASSOCIACÁA BRASILEIRADE
AGUAS SUBTERRANEAS www.abas.org

\title{
VULNERABILIDADE DOS AQUÍFEROS CÁRSTICOS SAPUCARI E MARUIM, BACIA SEDIMENTAR DE SERGIPE-ALAGOAS
}

\section{VULNERABILITY OFSAPUCARI AND MARUIM KARST AQUIFERS, SEDIMENTARY BASIN OF SERGIPE-ALAGOAS}

\author{
Daniela Dantas de Menezes Ribeiroㅜㅜ Sergio Augusto de Morais Nascimento²; \\ Antonio Jorge Vasconcellos Garcia ${ }^{3}$
}

Artigo recebido em: 23/05/2016 e aceito para publicação em: 04/09/2016.

DOI: http://dx.doi.org/10.14295/ras.v30i3.28634

\begin{abstract}
Resumo: Os aquíferos cársticos Sapucari e Maruim são importantes reservas hídricas, responsáveis pelo abastecimento dos municípios de sua abrangência e da grande Aracaju/SE. Os aquíferos cársticos possuem interesse notável, uma vez que as rochas carbonáticas ocupam extensas áreas, com características hidrológicas distintas de grande importância por suas reservas, vazões e qualidade da água. O trabalho teve como objetivo gerar o mapa de vulnerabilidade à contaminação dos aquíferos cársticos com aplicação do método EPIK e identificar áreas suscetíveis à contaminação das suas águas. Este método considera 4 parâmetros: Desenvolvimento de Epicarste (E); Importância da Cobertura de Proteção (P); Condições de Infiltração (I); e Desenvolvimento de rede Cárstica (C). As classes de vulnerabilidade apresentadas com a aplicação do método EPIK foram: muito alta, alta e moderada. As áreas com ocorrências de cavernas e feições cársticas como karrens e dolinas apresentaram os maiores valores de vulnerabilidade. As principais atividades potencialmente contaminantes identificadas na área foram: cultivos agrícolas, exploração mineral do calcário, indústria petroquímica, aglomerados urbanos, postos de gasolina e presença dos campos petrolíferos. Dados qualitativos de águas subterrâneas indicaram a presença de contaminação por óleos e graxas em dois poços nos municípios de Laranjeiras e Nossa Senhora do Socorro oriunda de postos de combustíveis, entre outros.
\end{abstract}

Palavras-chave: Epik. Vulnerabilidade. Aquíferos Cársticos. Bacia Sergipe-Alagoas.

\begin{abstract}
The karst aquifers Sapucari and Maruim are important water resources, responsible for human supply in the cities studied and metropolitan region of Aracaju/SE. The karst aquifers have great interest since the carbonate rocks occupy extensive areas, with distinct hydrological characteristics of great importance for their water reserve, pumping rate and water quality. The study aimed to generate the vulnerability map to contamination of karst aquifers with EPIK method application and identify areas susceptible to water contamination. This method takes into account four parameters: Development Epikarst (E); Protective Cover (P); Infiltration conditions (I); Karst network (K). The vulnerability classes presented with the EPIK method application were: very high, high and moderate. The areas with occurrences of caves, sinkholes and karrens showed the highest vulnerability values. The main potentially contaminating activities identified in the area were: agriculture activities, limestone explotation, petrochemical industry, urban development, gas stations and the presence of oil fields. Groundwater qualitative data indicated the presence of contamination by oil and grease from two wells in the cities of Laranjeiras and Nossa Senhora do Socorro originating from gas stations.
\end{abstract}

Keywords: EPIK. Vulnerability. Karst Aquifers. Sergipe-Alagoas Basin.

\section{INTRODUÇÃO}

As regiões cársticas possuem interesse econômico e hidrogeológico por suas significativas reservas de água subterrânea e ocorrências minerais. Tendo em vista a importân-

1 Universidade Federal da Bahia. Programa de Pós-Graduação em Geologia (UFBA) - E-mail: danielaprogeo@gmail.com

2 Universidade Federal da Bahia. Professor Associado (UFBA). E-mail: sergiomn@ufba.br

3 Universidade Federal da Bahia. Professor Associado (UFS). E-mail: garciageo@hotmail.com 
cia dos aquíferos cársticos para o abastecimento de água das populações, irrigação e indústria, tornam-se imprescindíveis ações de melhorias na gestão desses recursos hídricos, uma vez que cerca de $22 \%$ do abastecimento da Grande Aracaju (MENESES, 2015) provêm desses aquíferos cársticos.

Os mapas de vulnerabilidade e de risco auxiliam em atividades que objetivem a proteção das áreas de recarga e, consequentemente, preservem a boa qualidade das águas, frente às limitações técnicas e financeiras decorrentes dos trabalhos de remediação de possíveis contaminações. Além disso, permitem a compreensão dos diferentes graus de fragilidade dos aquíferos, sendo utilizado como ferramenta de gestão para o desenvolvimento local e regional.

No estudo e mapeamento da vulnerabilidade de aquíferos fraturados associados ao sistema Aquífero Serra Geral (REGINATO e AHLERT, 2013) foram empregados os métodos DRASTIC (ALLER et al., 1987) e GOD (FOSTER e HIRATA, 1988). O primeiro método utiliza sete parâmetros para análise da vulnerabilidade: D (profundidade do nível de água), R (recarga do aquífero), A (litologia do meio aquífero), $\mathrm{S}$ (tipo de solo), $\mathrm{T}$ (declividade), I (natureza da zona não saturada) e C (condutividade hidráulica). Para aplicação do método DRASTIC são atribuídos pesos de 1 a 10 a cada parâmetro. Estes são multiplicados por fatores que variam de 1 a 5 , definidos no método com base na relevância de cada parâmetro, para a avaliação da vulnerabilidade.

O método GOD, também aplicado no estudo para determinação das áreas do aquífero Bauru, no município de Rio Verde, estado de Goiás (PEREIRA JÚNIOR et al., 2015), emprega os parâmetros Grau de confinamento do aquífero (G), Substrato litológico (O) e Distância da água (D), na avaliação da vulnerabilidade. Atribui-se pesos de 0 a 1 para cada um dos parâmetros, o produto entre essas variáveis determina o índice de vulnerabilidade.

Os principais métodos aplicados na avaliação da vulnerabilidade de aquíferos são: DRASTIC, GOD, TPE, COP, PI, dentre outros (HIRATA e REBOUÇAS, 2001). Outros métodos para estimativa da vulnerabilidade de aquíferos também se destacam, como, por exemplo, o EPIK (DOERFLIGER et al.,1997).

O método EPIK foi desenvolvido para estimar a vulnerabilidade de aquíferos cársticos e é baseado nas características associadas ao fluxo e transporte no aquífero: Epicarste (E), Importância da Cobertura de Proteção (P),Condições de Infiltração (I) e Desenvolvimento de rede cárstica (K). Estudos realizados por Barrocu et al. (2007) e Gogu e Dassargues (2000) avaliaram o método EPIK na determinação da vulnerabilidade de aquíferos cársticos na região cárstica de "Supramonte" (Sardenha, Itália) e sul da Bélgica.

Os principais riscos para as águas subterrâneas na região estão relacionados a exploração mineral, agricultura, pastagens, presença de fábricas de cimento, indústria petroquímica, postos de combustíveis, campos de petróleo e, principalmente, os aglomerados urbanos.

Assim, o presente artigo tem como objetivo determinar a vulnerabilidade à contaminação dos aquíferos cársticos Sapucari e Maruim com aplicação do método EPIK. Propõese identificar áreas susceptíveis a contaminação, relacionando a vulnerabilidade com a presença de atividades potencialmente contaminantes, estabelecendo-se, consequentemente, os riscos para os citados aquíferos.

\section{LOCALIZAÇÃO E CARACTERIZA- ÇÃO DA ÁREA}

A área de estudo possui $345 \mathrm{~km}^{2}$, e situa-se a $30 \mathrm{~km}$ de Aracaju/SE. Engloba total ou parcialmente os municípios de Nossa Senhora do Socorro, Laranjeiras e Maruim (Figura 1). O acesso a área de estudo se dá pela BR-101 sentido Aracaju-Maceió, entroncamentos com as rodovias estaduais SE-090 e SE-245.

A área de estudo encontra-se representada pelo intervalo estratigráfico das formações Cotinguiba e Riachuelo, de idade cretácica, da Bacia Sedimentar de Sergipe-Alagoas. A Formação Cotinguiba é dividida em dois membros, o Aracaju e o Sapucari. O 
Membro Aracaju é constituído por calcilutitos, folhelhos e margas, enquanto que o Membro Sapucari, predominante na área de estudo, é caracterizado por calcilutitos maciços e brechóides (CAMPOS NETO et al., 2007).

A Formação Riachuelo é constituída por uma sequência sedimentar com predominância de clásticos na base e carbonatos no topo. A litologia permite individualizar na sub-bacia de Sergipe, os Membros Taquari, Maruim e Angico. O Membro Taquari é constituído por calcilutitos e folhelhos, o Membro Maruim é formado por calcarenito e calcirrudito oncolítico. Já o Membro Angico é constituído por arenito branco fino a conglomerático (CAMPOS NETO et al., 2007).

Sobrepondo-se a estes litotipos, encontra-se a Formação Barreiras, constituída por clásticos continentais finos a grosseiros, de coloração variada e grau de compactação insignificante (FEITOSA et al., 1998).

As feições estruturais regionais da sub-bacia sedimentar de Sergipe (Bacia SEAL) limitam-se por falhas normais. Estas possuem direções que acompanham o trend geral da bacia, $\mathrm{N} 45^{\circ} \mathrm{E}$, e são típicas de um "rift" gerado por distenção NO-SE (LANA, 1990). Trends secundários ocorrem em direções NW-SE, N-S e N-W.

O carste possui características hidrogeológicas e geomorfológicas distintas e grandes heterogeneidades espacial e temporal. O desenvolvimento da dissolução nesses terrenos depende de aspectos hidrodinâmicos e físico-químicos, como: armazenamento e circulação das águas subterrâneas condicionados à dissolução, fraturamentos e outras descontinuidades das rochas carbonáticas.

Uma ampla variedade de formas cársticas é produzida nesses ambientes. Os epi- carstes são feições cársticas de superfície, formas de dissolução do topo rochoso, dos tipos “karren” e dolinas (LOUCKS, 1999). As feições cársticas de superfície são descontínuas, ao longo da faixa carbonática analisada, concentrando-se as maiores ocorrências nas áreas dos calcários Sapucari.

Zonas com feições cársticas de subsuperfície são encontradas em descrição de poços tanto no Membro Sapucari como no Maruim, associadas a sistemas de fraturas regionais com formação de condutos e cavernas com variação em dimensão vertical de 3 a 10 metros e ocorrências na profundidade estratigráfica entre 10 e 140 metros.

Segundo Carvalho Junior (2005), nos terrenos das rochas carbonáticas representativas das formações Riachuelo e Cotinguiba, observam-se a presença frequente de feições de dissolução de topo rochoso de calcários, na forma de karrens arredondados, dolinas isoladas e fendas de dissolução nas cavas das pedreiras no município de Laranjeiras e em cortes de vertentes ao longo da BR-101.

Os condutos cársticos são descritos em fichas de poços de captação de águas subterrâneas em diferentes profundidades do perfil estratigráfico. Encontram-se associados aos sistemas de fraturas, direção NE, nas áreas da floresta do Ibura (Povoado da Estiva), município de Nossa Senhora do Socorro, povoados Mussuca e Cedro, no município de Laranjeiras e nas proximidades das pedreiras Brejo e Carapeba neste mesmo município.

Na área de ocorrência do Membro Sapucari ocorrem setores com um sistema de condutos com padrões em rede, composto por ramos principais na direção N15W, N40W e E-W (CARVALHO JUNIOR, 2005). 

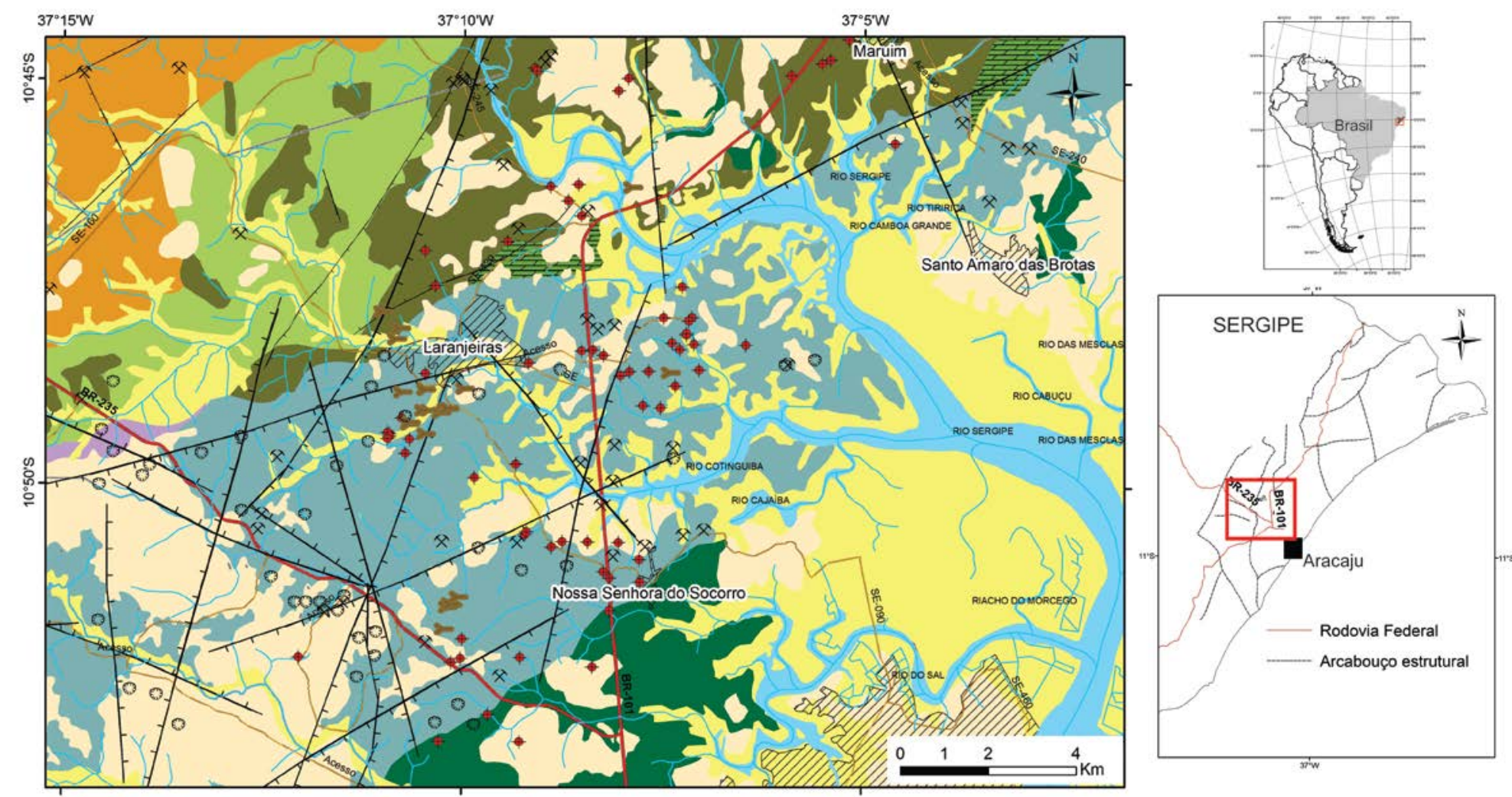





Figura 1 - Mapa de localização com áreas de ocorrências de feições cársticas, de rochas carbonáticas e distribuição de poços

Figure 1 - Location map with areas of karst features occurrences, carbonate rocks, and wells distribution 
Feições do tipo karren com caneluras (Figura 2) são observadas em afloramentos do município de Laranjeiras. Tubos de dissolução são observados no paredão da rocha, indicando o processo de formação de condutos (Figura 2).



Figura 2 - Feições do tipo karren com caneluras observadas em pedreira do Membro Sapucari, Fm. Cotinguiba, no município de Laranjeiras, estado de Sergipe

Figure 2 - Features of karren type with fluting observed in Sapucari Member quarry, Fm. Cotinguiba, city of Laranjeiras, State of Sergipe

As dolinas, depressões fechadas (Figura 3), ocorrem nas áreas próximas às drenagens do rio Cotinguiba, por vezes associadas às falhas geológicas com direção principal NE e ocorrências de cavernas.

Com base em levantamento realizado pelo grupo Espeleológico Centro da Terra (CENTRO DA TERRA, 2014), a área de estudo possui ocorrências de feições cársticas, qualificadas como: cavernas, grutas e tocas. Sendo que destas, 17 encontram-se no município de Laranjeiras (Figuras 4 e 5), 7 no município de Nossa Senhora do Socorro e 5 no município de Maruim.

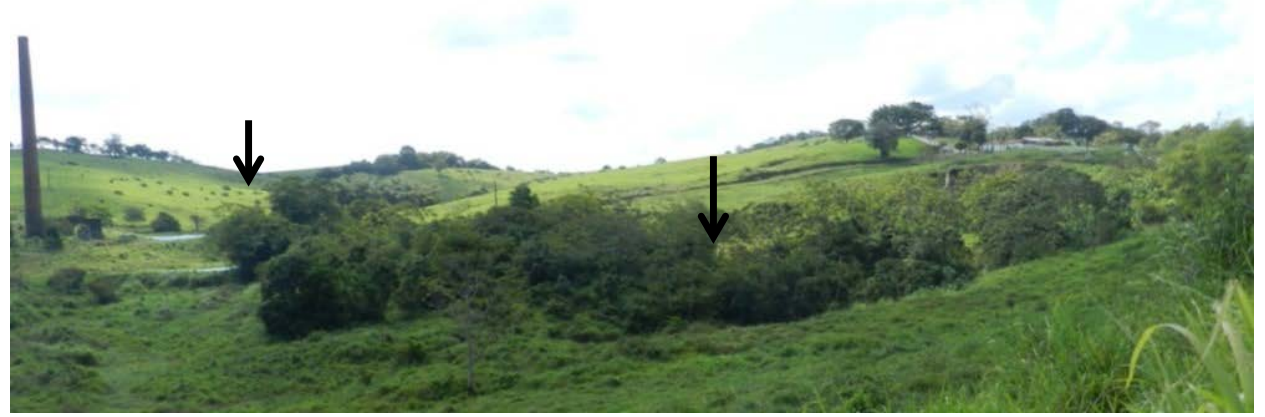

Figura 3 - Dolinas identificadas (setas em preto) na fotointerpretação geológica e georreferenciadas na fazenda Sergipe, município de Laranjeiras, representativa do Membro Sapucari da Fm. Cotinguiba

Figure 3 - Sinkholes identified (black arrows) in the geological photointerpretation at the Sergipe farm, city of Laranjeiras, representing the Sapucari Member, Fm. Cotinguiba 




Figura 4 - Gruta Matriana representativa dos calcários do Membro Maruim, Fm. Riachuelo, no município de Laranjeiras, Sergipe

Figure 4 - Matriana cave representative of the Maruim Member limestone, Fm. Riachuelo, city of Laranjeiras, State of Sergipe

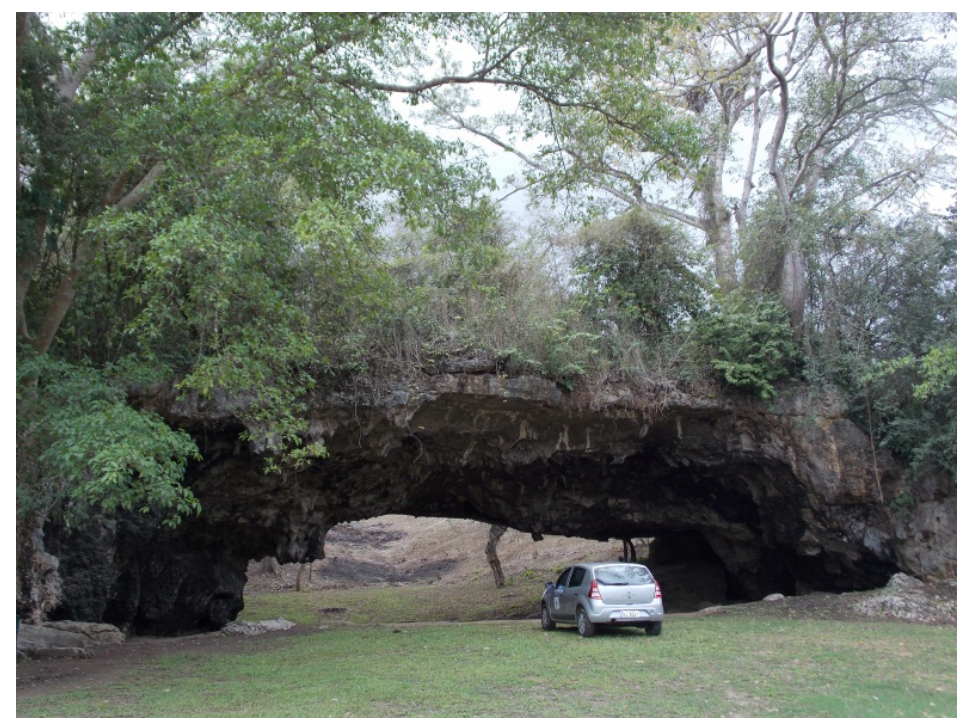

Figura 5 - Gruta da Pedra Furada representativa dos calcários do Membro Sapucari, Fm. Cotinguiba, município de Laranjeiras, Sergipe

Figure 5 - Pedra Furada cave representative of the Sapucari Member limestone, Fm. Cotinguiba, city of Laranjeiras, State of Sergipe

O aquífero Sapucari apresenta comportamento de aquífero cárstico, de alto gradiente hidráulico, e produtividade de maior destaque nos povoados Mussuca, Estiva e Machado nos municípios de Nossa Senhora do Socorro e Laranjeiras. A vazão média dos poços neste aquífero é de 54 $\mathrm{m}^{3} / \mathrm{h}$.
Os testes de produção obtidos das fichas de poços apresentam valor médio de transmissividade de $10^{-3} \mathrm{~m}^{2} / \mathrm{s}$. Este valor médio, bastante elevado, associado às ocorrências de fendas, referidas nas descrições litológicas, deixam pressentir também aqui a natureza cárstica do meio (FEITOSA et al., 1998). 
O aquífero Maruim também apresenta comportamento de aquífero cárstico, com maior produtividade no povoado $\mathrm{Pe}$ dra Branca, no município de Laranjeiras. A ocorrência de condutos nesse Membro é menor, apresentando uma evolução de sistema com pequenas cavernas (CARVALHO JUNIOR, 2005). A vazão média dos poços neste aquífero é de $25 \mathrm{~m}^{3} / \mathrm{h}$.

O fluxo subterrâneo no aquífero Maruim exibe feições típicas de circulação cárstica, desenvolvida ao longo de zonas fraturadas com geração de canais de magnitude não desprezível, evoluindo mesmo, em alguns casos, para cavernas (FEITOSA et al., 1998).

\section{MATERIAIS E MÉTODO}

O EPIK é um método utilizado na determinação da vulnerabilidade em aquíferos cársticos, desenvolvido por Doerfliger et al. (1997), que considera as características geológicas, geomorfológicas e hidrogeológicas, com destaque para o fluxo subterrâneo da área estudada.

O EPIK consiste na classificação e atribuição de pesos em multi-parâmetros, com sobreposição de dados geoespaciais e geração de um índice de vulnerabilidade das águas subterrâneas. São utilizados quatro parâmetros/atributos: Epicarste (E); Cobertura de Proteção (P); Condição de Infiltração (I); Desenvolvimento de rede cárstica (K). Um multiplicador é atribuído a cada parâmetro, refletindo a relativa importância ponderada.

As classes presentes em cada parâmetro recebem valores e são multiplicadas pelo respectivo peso e, em seguida, os produtos (mapas) são somados para se chegar a um valor final. Quanto maior a pontuação, maior é o grau de proteção da área, ou seja, menos vulnerável. No fim, o intervalo de pontuação numérica final é atribuído a classes de diferentes graus de vulnerabilidade. Os parâmetros EPIK são descritos da seguinte forma:

Epicarste (E): corresponde à zona de epicarste sob o solo consolidado. Baseia-se em estudos das formas cársticas presentes na área. Este parâmetro (Quadro 1) apresenta um alto nível de dificuldade para determinação das zonas de epicarste e conexão com rede cárstica (DOERFLIGER et al., 1997).

Com base nas ortofotocartas dos municípios litorâneos, escala 1:10.000 (SEPLAN-SE, 2003), foram realizados procedimentos de fotointerpretação geológica para identificação de fotolineamentos e análise morfológica. Esta última com o objetivo de identificar as feições cársticas dolinas e relações com ocorrências de cavernas, exploração mineral e fluxo subterrâneo.

Quadro 1 - Classes para o atributo Epicarste (Adaptado de DOERFLIGER et al., 1997)

Chart 1 - Classes for Epikarst attribute (Adapted from DOERFLIGER et al., 1997)

\begin{tabular}{|l|l|l|}
\hline Epicarste & $\mathrm{E}_{1}$ & \begin{tabular}{l} 
Morfologia cárstica- características \\
\hline Alto desenvolvimento \\
Indica a situação de maior vulnerabilidade, associada a ocor- \\
rência de depressões com acumulo de água, dolinas, karrens, \\
afloramentos com alta densidade de fraturas, por vezes relaci- \\
onados as falhas geológicas e arcabouço estrutural.
\end{tabular} \\
\hline $\begin{array}{l}\text { Moderado desenvolvi- } \\
\text { mento }\end{array}$ & $\mathrm{E}_{2}$ & $\begin{array}{l}\text { Correspondem as zonas intermediárias, em alinhamentos de } \\
\text { dolinas e vales secos. }\end{array}$ \\
\hline Pequeno ou ausente & $\mathrm{E}_{3}$ & $\begin{array}{l}\text { Sem fenômeno de morfologia cárstica e baixa densidade de } \\
\text { fraturas. Interseções de lineamentos identificados nas fotogra- } \\
\text { fias aéreas ou de imagens de satélite consistem em zonas alta- } \\
\text { mente fraturadas. Estas quando associadas as características tí- } \\
\text { picas geomorfológicas, podem ser mapeadas como E2. }\end{array}$ \\
\hline
\end{tabular}


Cobertura de Proteção (P): determinado a partir de mapas de uso da terra, de solos e fichas de descrição de poços de captação de águas subterrâneas (descrição de perfis litológicos).

Este parâmetro inclui tanto o solo (profundidade) como outras configurações geológicas, tais como: depósitos do quaternário ( silte, sedimentos inconsolidados e detritos), e outras camadas não-cársticas, por exemplo, argila e areia (Quadro 2).

Foram utilizados dados de fichas de poços de captação de águas subterrâneas. Informações como profundidade e características do solo (argiloso, arenoso) foram extraídas das fichas de poços, sobrepostos ao mapa de solos da área de estudo. Foram consultadas 50 fichas para determinação da profundidade do solo.

Quadro 2 - Classes para o atributo Cobertura de Proteção (Adaptado de DOERFLIGER et al., 1997)

Chart 2 - Classes for Protective Cover attribute (Adapted from DOERFLIGER et al., 1997)

\begin{tabular}{|c|c|c|c|}
\hline \multicolumn{2}{|c|}{$\begin{array}{l}\text { Cobertura de Pro- } \\
\text { teção }\end{array}$} & \multicolumn{2}{|l|}{ Características } \\
\hline \multirow[t]{5}{*}{ Ausente } & & $\begin{array}{l}\text { A. Solo cobrindo diretamente ro- } \\
\text { chas calcárias ou rochas com } \\
\text { alta permeabilidade (camadas } \\
\text { de detritos grosseiros, ex. frag- } \\
\text { mentos detritos). }\end{array}$ & $\begin{array}{l}\text { B. Solo cobrindo camadas geológicas } \\
\text { de baixa permeabilidade, ex. silte, } \\
\text { argilas e folhelhos. }\end{array}$ \\
\hline & $\mathrm{P}_{1}$ & 0-20 cm de solo & $\begin{array}{l}0-20 \mathrm{~cm} \text { de solo em camadas com espes- } \\
\text { sura menor que } 1 \text { metro. }\end{array}$ \\
\hline & $\mathrm{P}_{2}$ & $20-100 \mathrm{~cm}$ de solo & $\begin{array}{l}20-100 \mathrm{~cm} \text { de solo com camadas de es- } \\
\text { pessura menor que } 1 \text { metro. }\end{array}$ \\
\hline & $\mathrm{P}_{3}$ & $100-200 \mathrm{~cm}$ de solo & $\begin{array}{l}\text { Solo }>100 \mathrm{~cm} \text { cobrindo camadas com } \\
\text { baixa permeabilidade. }\end{array}$ \\
\hline & $\mathrm{P}_{4}$ & $>200 \mathrm{~cm}$ & $\begin{array}{l}\text { Solo }>\text { que } 100 \mathrm{~cm} \text { cobrindo depósitos de } \\
\text { permeabilidade muito baixa ou aquiclu- } \\
\text { des }>8 \text { metros de espessura. }\end{array}$ \\
\hline
\end{tabular}

Condições de Infiltração: baseado na identificação de zonas de concentração da infiltração. Trata do tipo de recarga do aquífero cárstico, não sendo considerada esta em termos de quantidade e localização. A recarga é classificada em difusa ou concentrada (descritas no Quadro 3).

Os dados necessários para a determinação das condições de infiltração no método EPIK consistem em mapas de uso da terra e mapas topográficos (geração de modelo de terreno para a determinação da declividade). O mapa de Uso e Ocupação do Solo (SRH, 2014), escala 1:25.000, foi utilizado na determinação das condições de infiltração, a partir da análise e classificação dos principais usos da terra com base na metodologia EPIK.

O mapa de uso da terra foi sobreposto ao mapa de declividade, elaborado a partir dos dados de curvas de nível, presentes nas ortofotocartas (SEPLAN-SE, 2003) e do modelo digital de elevação, gerado através da imagem ASTER (Advanced Spaceborne Thermal Emission and Reflection Radiometer), com resolução espacial de $30 \mathrm{~m}$. 
Quadro 3 - Classes para o atributo Condições de Infiltração (Adaptado de DOERFLIGER et al., 1997) Chart. 3 - Classes for Infiltration Conditions attribute (Adapted from DOERFLIGER et al., 1997)

\begin{tabular}{|l|l|l|}
\hline Condições de Infiltração & Características \\
\hline Concentrada & $\mathrm{I}_{1}$ & $\begin{array}{l}\text { Riachos perenes ou temporários- alimentando dolinas e sumi- } \\
\text { douros - áreas de captação de água dos rios mencionados, in- } \\
\text { cluindo sistemas de drenagens naturais e artificiais. }\end{array}$ \\
\cline { 2 - 4 } & $\mathrm{I}_{2}$ & $\begin{array}{l}\text { Áreas de captação de água em riachos } \mathrm{I}_{1} \text { (sem sistema de dre- } \\
\text { nagem artificial) com declividade superior a 10\% para áreas } \\
\text { cultivadas e 25\% para pastagens. }\end{array}$ \\
\cline { 2 - 4 } & $\mathrm{I}_{3}$ & $\begin{array}{l}\text { Áreas de infiltração concentrada (sem sistema de drenagem ar- } \\
\text { tificial) declividade }<10 \% \text { em campos cultivados e }<25 \% \text { em } \\
\text { campos de pastagens. }\end{array}$ \\
\cline { 2 - 4 } Difusa & $\mathrm{I}_{4}$ & Terras remanescentes \\
\hline
\end{tabular}

\section{Desenvolvimento de rede cárstica (K):}

O desenvolvimento da rede cárstica e seu grau de organização possui grande influência na velocidade do fluxo da água (Quadro 4). Como o mapeamento detalhado de redes cársticas não é possível na maioria dos casos, um único valor por captação é comumente usado (DOERFLIGER et al., 1997).

Para a determinação deste parâme- tro foram utilizados dados de poços de águas subterrâneas com identificação e profundidade de condutos e cavernas no perfil estratigráfico do poço. Cerca de 7 Poços apresentaram indicações de condutos e cavernas. Estes dados foram sobrepostos espacialmente, as ocorrências de cavernas já mapeadas em superfície, e zonas de falhas geológicas, com o objetivo de identificar o desenvolvimento da rede cárstica.

Quadro 4 - Classes para o atributo Rede Cárstica (Adaptado de DOERFLIGER et al., 1997)

Chart 4 - Classes for Karst Network attribute (Adapted from DOERFLIGER et al., 1997)

\begin{tabular}{|l|l|l|}
\hline Rede cárstica & $\mathrm{K}_{1}$ & \begin{tabular}{l} 
Caracterização \\
\hline Rede bem desenvolvida \\
volvida com condutos em decímetro a metro com \\
pouca obstrução e interconectados.
\end{tabular} \\
\hline $\begin{array}{l}\text { Rede cárstica pouco desenvol- } \\
\text { vida }\end{array}$ & $\mathrm{K}_{2}$ & $\begin{array}{l}\text { Rede pouco desenvolvida; drenagem pouco desen- } \\
\text { volvida e condutos em decímetros ou menores. }\end{array}$ \\
\hline Aquifero fissural ou misto & $\mathrm{K}_{3}$ & $\begin{array}{l}\text { Surgências emergindo do meio poroso do terreno. } \\
\text { Aquíferos fissurais, não cársticos. }\end{array}$ \\
\hline
\end{tabular}

Os quatros parâmetros são ponderados e, posteriormente, classificados. No ambiente SIG (Sistema de Informações Geográficas), são gerados os mapas representativos de cada parâmetro com seus pesos respectivos.
A Figura 6 apresenta os procedimentos de classificação e sobreposição de mapas, realizados no ArcGis 10.2, para a geração do mapa de vulnerabilidade de aquíferos cársticos. 


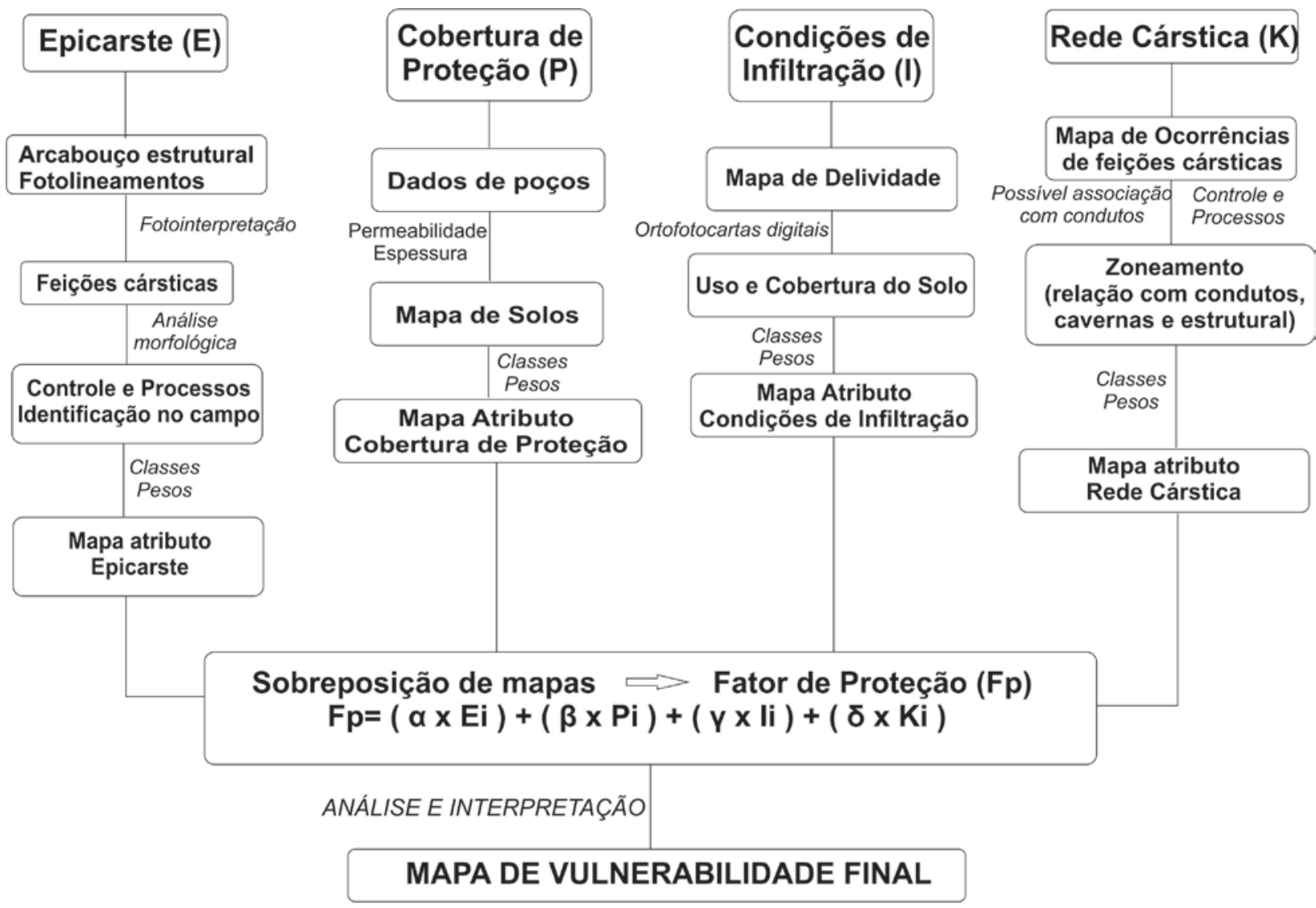

Figura 6 - Fluxograma metodológico de procedimentos realizados em Ambiente SIG para criação do mapa de vulnerabilidade (método EPIK) dos aquíferos cársticos Sapucari e Maruim, Sub-bacia de Sergipe, Bacia SEAL

Figure 6 - Flowchart of procedures performed in GIS environment to create the vulnerability map (EPIK method) of the Sapucari and Maruim karst aquifers, Sub-basin of Sergipe, Basin of SEAL

O Quadro 5 apresenta os pesos atribuídos a cada parâmetro EPIK, onde o menor valor indica maior sensibilidade do aquífero. $\mathrm{O}$ pectivos pesos para cada parâmetro EPIK, sendo o menor valor indicativo de maior Quadro 6 apresenta as classes e seus ressusceptibilidade.

Quadro 5 - Pesos relativos atribuídos aos parâmetros analisados pelo método EPIK. Quanto mais baixo o valor numérico, maior é a susceptibilidade a contaminação (Adaptado de DOERFLIGER et al., 1997)

Chart 5 - Relative weights assigned to the parameters analyzed by the EPIK method. The lower the numerical value, the greater the susceptibility to contamination (Adapted from DOERFLIGER et al., 1997)

\begin{tabular}{|l|l|l|l|}
\hline Epicarste (E) & Cobertura de Proteção (P) & Condições de Infiltração (I) & Rede cárstica (K) \\
\hline$\alpha$ & $\beta$ & $\gamma$ & $\delta$ \\
\hline 3 & 1 & 3 & 2 \\
\hline
\end{tabular}


Quadro 6 - Pesos das classes para os parâmetros E, P, I e K. Quanto menor o valor, maior a susceptibilidade a contaminação (Adaptado de DOERFLIGER et al.,1997)

Chart 6 - Classes weights of the parameters E, P, I and K. As smaller is the value greater the susceptibility to contamination (Adapted from DOERFLIGER et al., 1997)

\begin{tabular}{|l|l|l|l|l|l|l|l|l|l|l|l|l|l|l|}
\hline \multicolumn{3}{|c|}{ Epicarste } & \multicolumn{3}{c|}{ Cobertura de Proteção } & \multicolumn{3}{c|}{ Condições de Infiltração } & \multicolumn{3}{|c|}{ Rede Cárstica } \\
\hline $\mathrm{E}_{1}$ & $\mathrm{E}_{2}$ & $\mathrm{E}_{3}$ & $\mathrm{P}_{1}$ & $\mathrm{P}_{2}$ & $\mathrm{P}_{3}$ & $\mathrm{P}_{4}$ & $\mathrm{I}_{1}$ & $\mathrm{I}_{2}$ & $\mathrm{I}_{3}$ & $\mathrm{I}_{4}$ & $\mathrm{~K}_{1}$ & $\mathrm{~K}_{2}$ & $\mathrm{~K}_{3}$ \\
\hline 1 & 3 & 4 & 1 & 2 & 3 & 4 & 1 & 2 & 3 & 4 & 1 & 2 & 3 \\
\hline
\end{tabular}

O fator de proteção Fp é calculado com a equação (1), e tem como objetivo representar um modelo hidrogeológico simplificado. O índice final de vulnerabilidade apresenta as seguintes classes: muito alta (9-19); alta (20-25); moderada (>25); baixa (> 25 com a presença das categorias P4 +I3, I4). As Zonas de Proteção (S) são definidas em: S1 (vulnerabilidade muito alta); S2 (alta vulnerabilidade); S3 (moderada vulnerabilidade).

Equação 1 (DOERFLIGER et al., 1997):

$F p=\left(\alpha X E_{i}\right)+\left(\beta X P_{i}\right)+\left(\gamma X_{i}\right)+\left(\delta X K_{i}\right)$

Onde:

Fp - índice de proteção

$\alpha ; \beta ; \gamma ; \delta$ - Pesos de cada parâmetro

$E_{\mathrm{i}} ; \mathrm{P}_{\mathrm{j}} ; \mathrm{I}_{\mathrm{k}} ; \mathrm{K}_{\mathrm{l}}$ - classes dos parâmetros.

\section{RESULTADOS OBTIDOS}

\section{Parâmetros de vulnerabilidade EPIK}

Epicarste (E): foram identificadas ocorrências de cavernas, dolinas e karrens com caneluras e pequenos condutos em formação nos afloramentos de pedreiras no municí- pio de Laranjeiras, como também fraturas identificadas nos afloramentos (com direcionamento preferencial NE-SW) e associações com falhas geológicas (Figura 7), que acompanham o trend geral da bacia, $\mathrm{N} 45^{\circ} \mathrm{E}$.

As áreas com maiores ocorrências das morfologias mencionadas foram classificadas como $\mathrm{E}_{1}$ (peso 1). Áreas com ocorrências moderadas foram classificadas como $\mathrm{E}_{2}$ (peso 3). Já as áreas com poucas ocorrências ou ausentes receberam a classe $\mathrm{E}_{3}$ (peso 4).

Cobertura de Proteção (P): neste parâmetro, a profundidade da cobertura de proteção (solo) foi determinada com base nos perfis estratigráficos de poços de captação de águas subterrâneas. Os poços apresentam variação de profundidade máxima de cobertura do solo entre 1 a 20 metros na área de estudo.

Com base na descrição de perfis de poços, o solo é de textura argilosa de cores amarelo e vermelho, por vezes, de textura arenosa com grão finos e bem arredondados. Sobreposto ao mapa de solos, o tipo correspondente predominante é o Argissolo Vermelho Amarelo, seguido do Gleissolo, Chernossolo e Espodossolo (SRH, 2014). 


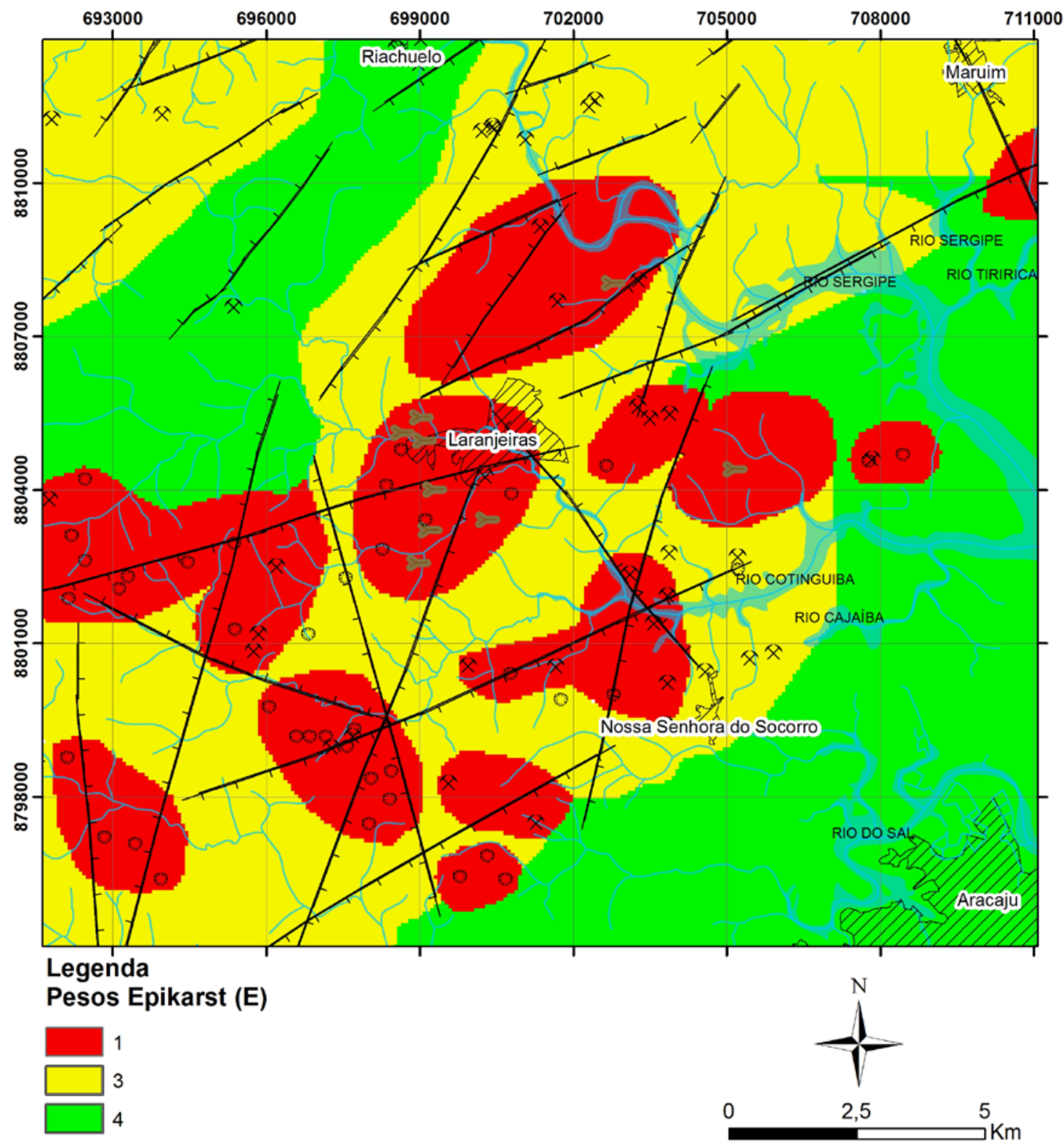

Convenções Geológicas

( A Afloramentos de Rochas Carbonáticas

2 Cavernas

Dolinas- fotointerpretação

VI/7 Sede municipal

Rede Hidrográfica Bifiliar

$\longrightarrow$ T Falhas Geológicas ( Falha Normal Provável)

Figura 7 - Mapa representativo do parâmetro Epicarste com distribuição de cavernas, dolinas e falhas geológicas associadas

Figure 7- Representative map of Epikarst parameter with distribution of caves, sinkholes and asso ciated faults

Na Figura 8, as zonas de peso 2 correspondem as ocorrências com menor cobertura de proteção (em torno de 1 metro).
Já as zonas de peso 3 e 4 possuem profundidade superior a 1 metro recobrindo solo com baixa permeabilidade. 
Condições de Infiltração (I): os principais usos da terra identificados na área de estudo são formados por: pastagens, cultivos agrícolas, solo exposto, área degradada, área industrial, floresta estacional, terras remanescentes e drenagens.

As áreas de drenagens associadas a ocorrências de dolinas e cavernas foram mapeadas como classe $\mathrm{I}_{1}$. As zonas com cultivos agrícolas, solo exposto, áreas degradadas e pastagens, próximas a riachos e nascentes, com declividade superior a $10 \%$ e 25\%, receberam classe I2. Áreas com cultivos agrícolas e declividade $<10 \%$, e $<25 \%$ em áreas de pastagens foram classificadas como I $I_{3}$. Já as terras remanescentes e floresta estacional foram classificadas como I 4 (Figura 9).

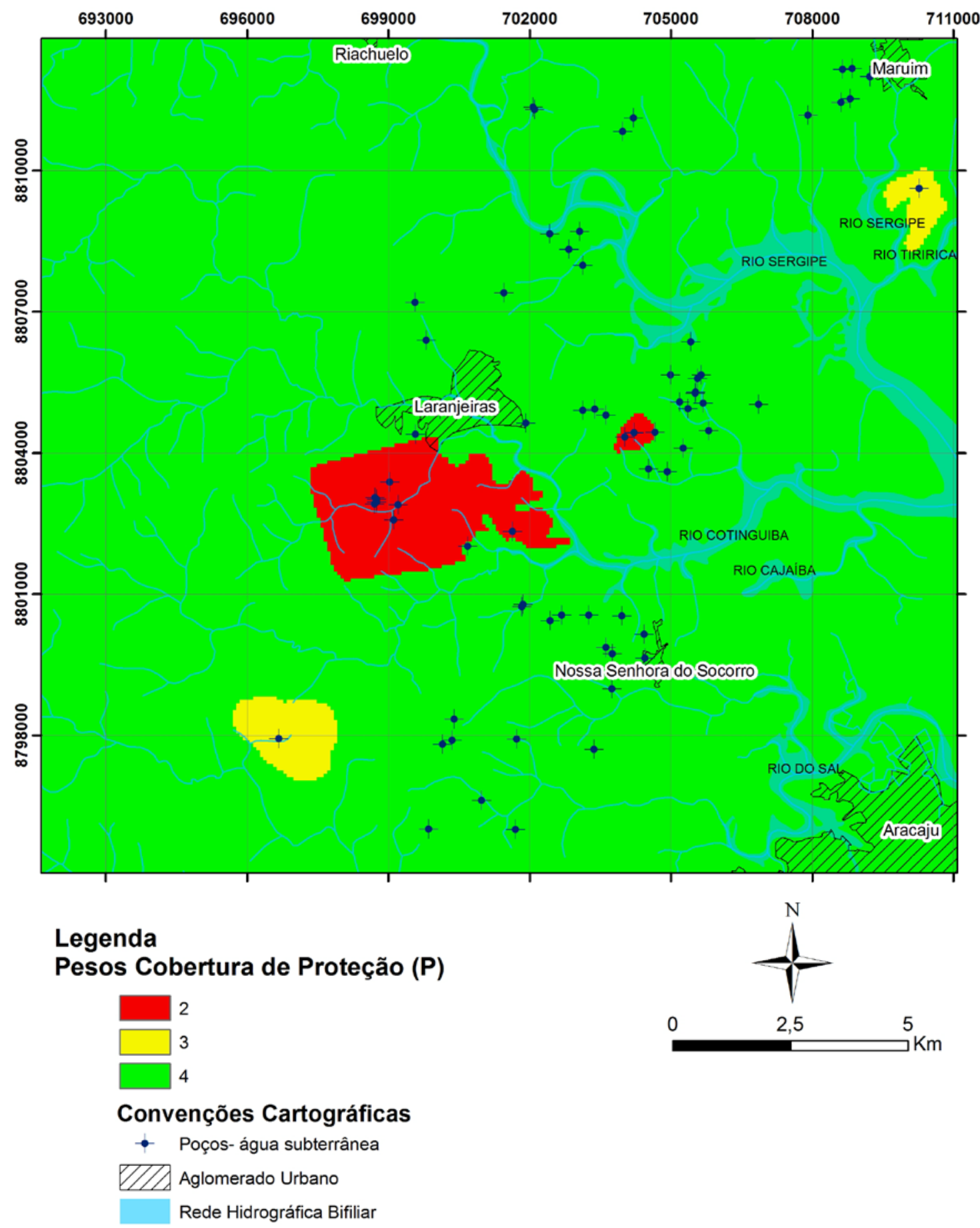

Figura 8 - Mapa representativo do parâmetro Cobertura de Proteção

Figure 8 - Representative map of Protection Cover parameter 




Figura 9 - Mapa representativo do parâmetro Condições de Infiltração

Figure 9- Representative map of Infiltration Conditions parameter

Rede cárstica (K): o processo de carstificação (dissolução) permite a geração de uma rede de condutos, desenvolvidos por conta da variação espacial da permeabilidade da 
rocha e potencial de infiltração, para os quais são direcionados os fluxos subterrâneos.

Aberturas com diâmetro entre 5-10 mm ou mais são consideradas verdadeiros condutos-cavernas (FORD, 1988). A rede de condutos pode ser mais ou menos bem desenvolvida e conectada, dependendo dos sistemas cársticos analisados.

As áreas de rede cárstica foram mapeadas a partir de dados de poços de captação de águas subterrâneas. Foram encontrados condutos em profundidades de 1112 ; 16-20; 30-40; 44-50; 54-57; 134-136 metros. Os condutos estão associados às grandes falhas da bacia com direcionamento preferencial NE-SW, distribuídos espacialmente em diferentes pontos da área de estudo.

Ocorrências de cavernas também foram identificadas em profundidades com variação entre de 19-23 e 100 metros em três poços na região dos povoados Mussuca (C) e Estiva (A) (Figura 10).

Como a distribuição dessas feições cársticas se deu em diferentes pontos da área estudada, optou-se pela atribuição da classe $\mathrm{K}_{1}$ (rede bem desenvolvida), de peso 1 , presença de rede cárstica moderada a bem desenvolvida, com condutos em decímetro a metro com pouca obstrução e interconectados a drenagens e falhas geológicas.

\subsection{Mapa de vulnerabilidade final da re- gião}

O mapa de vulnerabilidade EPIK foi gerado no SIG Arcgis $10.2{ }^{\circledR}$, com o auxílio da ferramenta "Spatial AnalystRaster Calculator”. Esta ferramenta é apli- cada no processo de sobreposição e álgebra de mapas, com uso de operadores como multiplicação e soma, com base na Equação 1, descrita no item Materiais e Método.

O fator de proteção apresentou variação de 10 a 30 , sendo que os valores mais baixos correspondem às áreas de maior vulnerabilidade. As classes de vulnerabilidade (Figura 10) apresentadas com a aplicação do método EPIK foram: muito alta $\left(71,80 \mathrm{~km}^{2}\right)$, alta $\left(107,01 \mathrm{~km}^{2}\right)$ e moderada $\left(174,42 \mathrm{~km}^{2}\right)$. As áreas de proteção, definidas com base no EPIK, estão associadas às áreas de maior vulnerabilidade, sendo assim classificadas: vulnerabilidade muito alta- área de proteção S1; alta- S2; e moderada- S3.

As zonas com ocorrências de cavernas e feições cársticas como karrens e dolinas, nos povoados Machado e Estiva, apresentaram os maiores valores de vulnerabilidade, classificada como área de proteção S1. As áreas de exploração de calcário apresentaram variação de vulnerabilidade muito alta a alta, sendo definidas como zonas de proteção S1 e S2.

As principais atividades potencialmente contaminantes consistem em: cultivos agrícolas, como a cana-de-açúcar, exploração mineral do calcário, indústrias, aglomerados urbanos, postos de gasolina e presença dos campos petrolíferos de Riachuelo e Carmópolis ao norte da área de estudo.

O mapa de vulnerabilidade sobreposto às áreas das atividades potencialmente contaminantes (Figura 11) indica riscos potenciais de contaminação nos povoados Machado (B), Estiva (A) e área da Floresta Ibura e adjacências (A). 

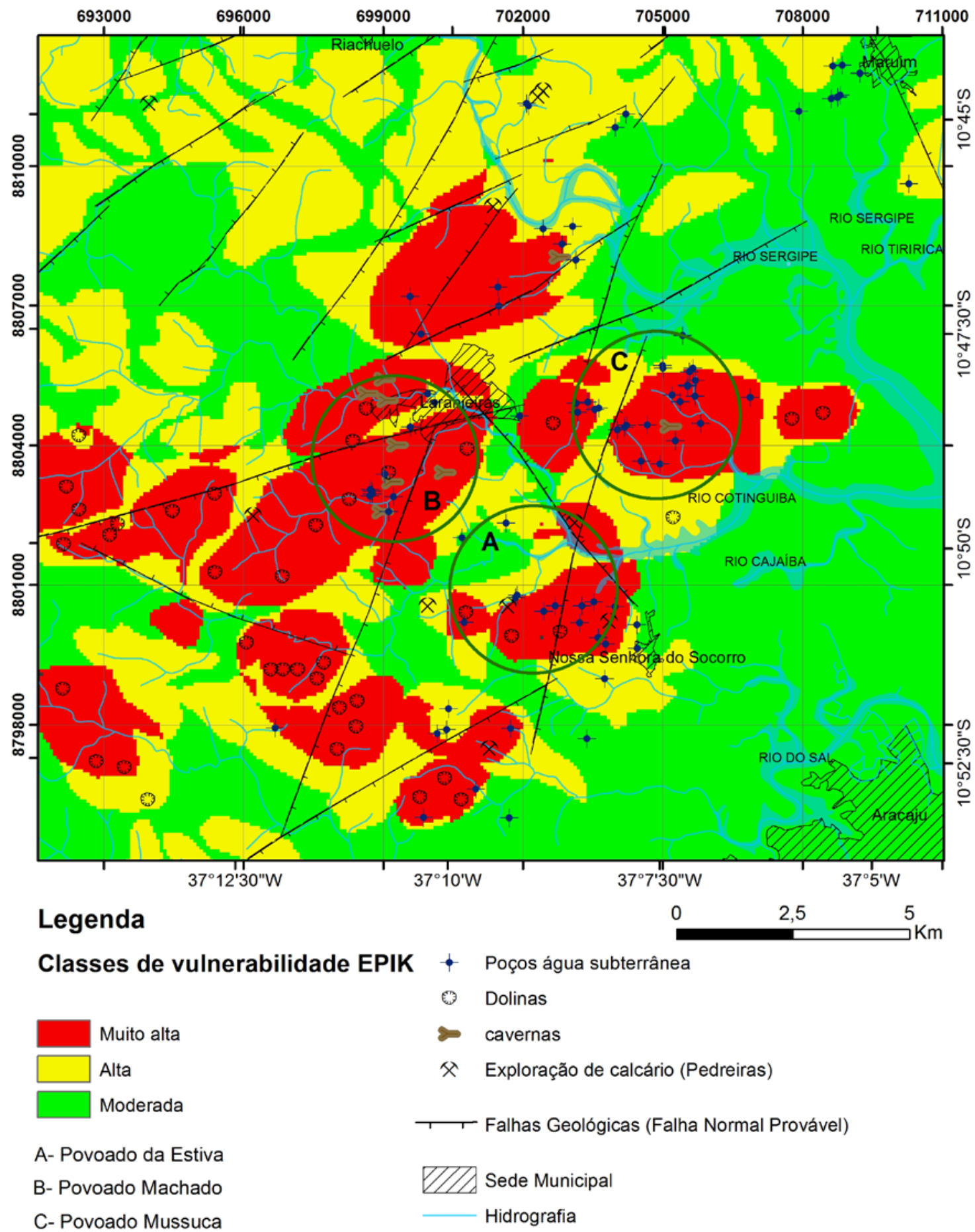

Figura 10 - Mapa de vulnerabilidade dos aquíferos cársticos Maruim e Sapucari com destaque para os povoados da Estiva (A), Machado (B) e Mussuca (C) de maior ocorrência de feições cársticas e exploração do calcário

Figure 10 - Vulnerability map of the Maruim and Sapucari karst aquifers with distinction to the Estiva (A), Machado (B) and Mussuca (C) villages of higher karst features occurrences and limestone prospection 


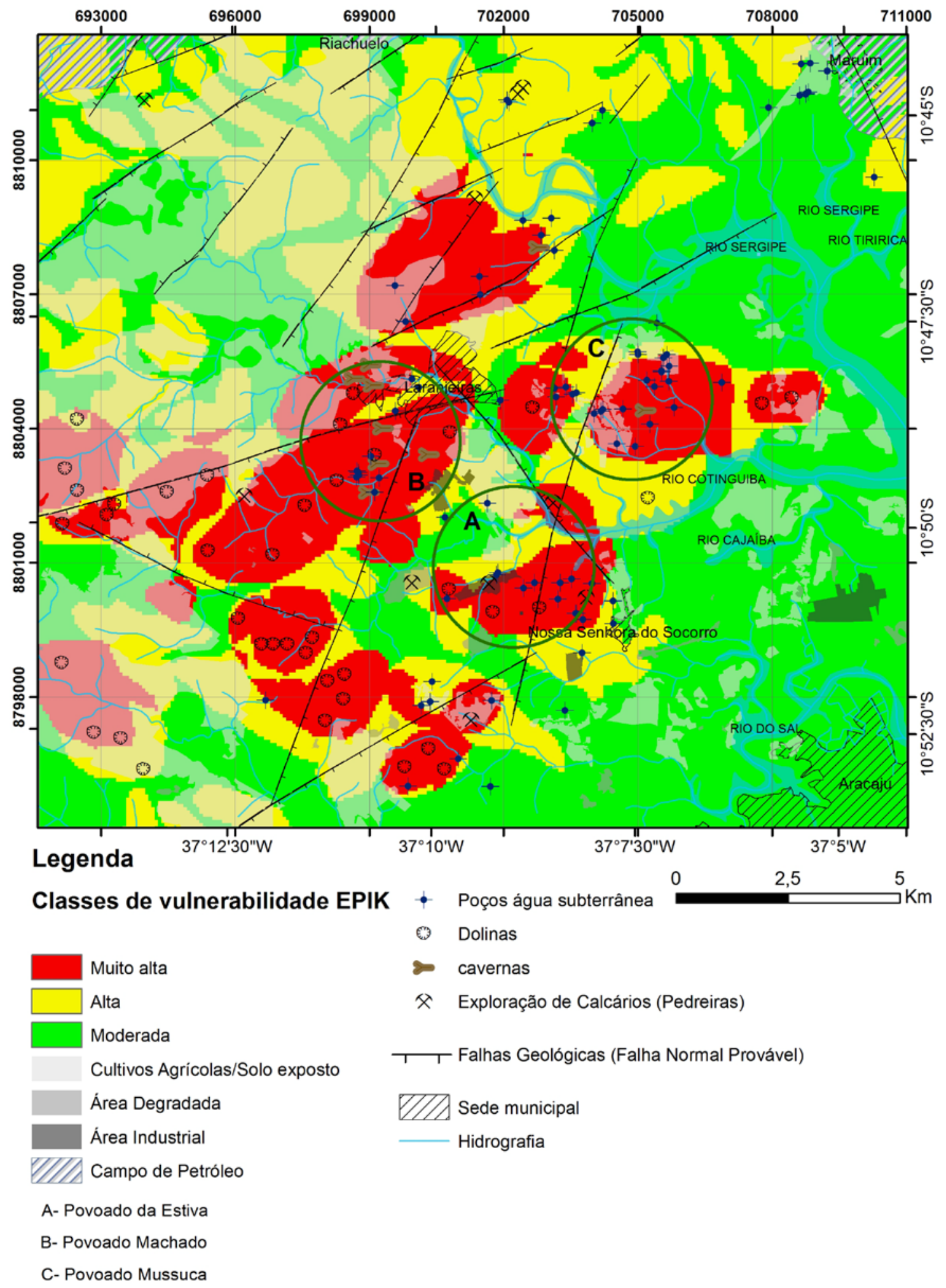

Figura 11 - Mapa de vulnerabilidade a contaminação de aquiferos com sobreposição das atividades potencialmente contaminantes presentes na área de estudo

Figure 11 - Vulnerability map with overlay of potentially contaminating activities present in the study area 


\section{CONCLUSÕES}

A aplicação do método EPIK permitiu a geração do mapa de vulnerabilidade dos aquíferos cársticos Maruim e Sapucari e a identificação de áreas potencialmente sujeitas à contaminação.

Os setores (A, B e C) apresentam maior vulnerabilidade à contaminação das águas subterrâneas, uma vez que há maiores ocorrências de feições cársticas e formação de pequenos condutos e fraturas em afloramentos analisados. Nesses, são observadas a presença de atividades de postos de combustíveis; exploração de calcário; indústrias de cimento e petroquímicas; cultivos agrícolas, principalmente da cana-deaçúcar; e adensamento urbano.

Análises químicas de águas subterrâneas indicaram a presença de contamina- ção por óleos e graxas em dois poços nos municípios de Laranjeiras e Nossa Senhora do Socorro (concentrações de 5,2 e 5,8 $\mathrm{mg} / \mathrm{l}$, respectivamente). As amostras coletadas permitiram identificar que a contaminação presente por óleos e graxas é oriunda de postos de combustíveis presentes nestas unidades.

Com base nesse mapa, foi possível visualizar a distribuição espacial da vulnerabilidade e suas diferentes classes, como também relacionar as diferentes zonas de proteção propostas pelo EPIK. O mapa de vulnerabilidade do sistema cárstico local permite um melhor entendimento para a definição de áreas de proteção ambiental desse aquífero de grande importância, responsável pelo abastecimento de água da Grande Aracaju.

\section{REFERÊNCIAS}

ALLER, L., LEHR, Jay H., PETTY, R. DRASTIC: A Standardized system of evaluating groundwater pollution potential using hidrogeologic settings. United States: EPA, 1987.

BARROCU, G.; MUZZU, M.; URAS, G. Hydrogeology and vulnerability map (Epik method) of the "Supramonte" karstic system, north-central Sardinia. Environment Geology, v.51, p.701-709, 2007.

CAMPOS NETO, O.P.A.; LIMA W.S.;CRUZ F.E.G. Bacia Sergipe-Alagoas. Boletim de Geociências da Petrobras, v.15, n. 2,p. 405-415, 2007.

CARVALHO JÚNIOR, A. L. P. Hidrogeologia e Processos Cársticos nas Formações Muribeca, Riachuelo e Cotinguiba na Bacia SE/AL em Sergipe. São Paulo, São Paulo, Brasil, 2005. 146 p. Tese (Doutoramento em Recursos Minerais e Hidrogeologia).Universidade de São Paulo.

CENTRO DA TERRA. Projeto Expedição Centro da Terra: Conhecendo as Cavernas no Meio Ambiente. Aracaju, 2014.

DOERFLIGER， N.; JEANNIN, P.; ZWAH
LEN, F. Water vulnerability assessment in karst environments: a new method of defining protection area susing a multi-attribute approach and GIS tools (EPIK method). Environmental Geology. International Journal of Geosciences, v. 39, n. 2, p.165-176, 2015.

FEITOSA, Edilton. C(Coord.); FILHO, J. M., DEMÉTRIO, J. G. A., SANTOS, R.Q., SANTOS, M. A. V., AGUIAR, N. F., DEMÉTRIO, J. A.Avaliação dos Aquíferos da Bacia Sergipe/Alagoas entre Aracaju e Capela. Recife: LABHID-Laboratório de Hidrogeologia CTG/UFPE.PETROBRAS, 175 p,1998.

Ford, D. C. Characteristics of dissolutional cave systems in carbonate rocks. SpringerVerlag, p. 25-57, 1988.

GOGU, R, C.; DASSARGUES, A. Sensitivity analysis for the EPIK method of vulnerability assessment in a small karstic aquifer, Southern Belgium.Hidrogeology Journal, v.8, p. 337345, 2000.

FOSTER, S. S. D.; HIRATA, R. C. A. Groundwater pollution risk evaluation: the methodology using available data. Lima: CEPIS/PAHO/WHO, 74 p, 1988. 
HIRATA, R. REBOUÇAS, A. C. La protección de los recursos hídricos subterráneos: Una visión integrada, basadaen perímetros de protección de pozos y vulnerabilidad de acuíferos. 2001. Disponível em: http://www.medioambienteonline.com/site/root/resour-

ces/technology/857.html. Acesso: 21 jun. 2010.

LANA, M. C. Bacia de Sergipe-Alagoas. Origem e Evolução de Bacias Sedimentares,p.311-332, 1990.

LOUCKS, R. G. Paleocave Carbonate Reservoirs: Origins, Burial-Depth Modifications, Spatial Complexity, and Reservoir Implications. AAPG Bulletin, v. 83, 1795-1834 p,1999.

MENESES, J.W de A. Recursos Hídricos no Estado de Sergipe. In: Semana de Geologia da UFS, 3., 2012. Palestra... São Cristóvão: UFS/DESO, 2012.
PEREIRA JUNIOR, L.C.; SOARES, L.T.; CASTRO, S. S. Vulnerabilidade Natural e Risco de Contaminação do Aquífero Bauru no Município de Rio Verde - GO. Águas Subterrâneas, v. 29, n.2, p. 129-145, 2015.

REGINATO, P. A. R.; AHLERT, S. Vulnerabilidade do Sistema Aquífero Serra Geral na Região Nordeste do Estado do Rio Grande do Sul. Águas Subterrâneas. V. 27, n.2, p. 32-46, 2013.

SECRETARIA DE ESTADO DO PLANEJAMENTO, ORÇAMENTO E GESTÃO DE SERGIPE- SEPLAN. Base Cartográfica dos Municípios Litorâneos, 2003.

SUPERINTENDÊNCIA DE RECURSOS HÍDRICOS DE SERGIPE (SRH). Atlas Digital de Recursos Hídricos do Estado de Sergipe. Aracaju, 2014. CD-Room. 